\title{
POLARIMETRIC TECHNIQUES FOR SAR CONTRAST AND RESOLUTION ENHANCEMENT
}

\author{
Carlos López Martinez ${ }^{1}$, Xavier Fàbregas Canovas ${ }^{1}$, Antoni Broquetas i Ibars ${ }^{2}$, and Ernst Krogager ${ }^{3}$ \\ ${ }^{1}$ Universitat Politècnica de Catalunya, Barcelona, Spain \\ ${ }^{2}$ Institut de Geomatica, Barcelona, Spain \\ ${ }^{3}$ Danish Defence Research Establishment, Copenhagen, Denmark
}

\section{INTRODUCTION}

In the last decades, Synthetic Aperture Radar systems, known as SAR, have become an important tool in Remote Sensing. This importance is due to different aspects that solve some of the problems that other systems designed to the same purpose have, like optical systems. One of the main features of the SAR systems is that they are "all-weather" systems, that is, the systems are almost independent of the weather conditions (clouds, rain, etc.). Also, the systems can take images during day and night. These features make possible to SAR systems to take information or images all the time, and almost all useable, whereas in the case of optical systems only about ten per cent of the images are not affected by weather conditions.

Also the systems are coherent as they acquire the amplitude and the phase of the returned signal. However, this coherent nature at the same time poses one of the main problems of the SAR systems: the speckle noise. This noise appears both on the amplitude and on the phase of the signal, but with different characteristics.

There are a lot of techniques that try to remove speckle noise from the data, but they are mainly applied on the amplitude information. There are also some techniques that try to remove speckle form phase. The techniques designed to remove speckle from the amplitude or from the phase are slightly different. For the amplitude, the main feature of the speckle noise is that it follows a multiplicative model over the desired data [1], then the techniques have to be adapted to it. The techniques that remove the speckle from the amplitude can be divided in multilook and spatial filtering techniques [1]. In the first case, uncorrelated data of the same place are obtained and then averaged. In the second case, the average is performed over the same image. These techniques remove speckle, but they have the problem of loss of resolution.

The techniques that are applied over the phase information have poor results compared with those obtained in the case of amplitude. Some techniques make a combination between the polarimetric channels giving only one image with the consequent loss of polarimetric information [2]. In other cases the intensities are restored using the idea of polarisation diversity [3]. There are also techniques that make a polarimetric speckle reduction [4] based on process the real and imaginary parts of the pola- rimetric channels. In this article a new way to remove speckle from amplitude and phase is presented. The main advantage of this algorithm is that both parts are processed almost in the same way.

\section{COHERENT DECOMPOSITION}

The concept of polarimetric decomposition is widely applied for processing polarimetric SAR data [5], [6], [7]. As an example of this, the coherent decomposition of a complex scattering matrix into three components shall be demonstrated. The decomposition is given by the following general representation [8],

$$
\begin{aligned}
{[S] } & =e^{j \varphi}\left\{e^{j \varphi_{s}} k_{s}[S]_{s p h e r e}\right. \\
& \left.+k_{d}[S]_{\text {diplanel } \theta}+k_{h}[S]_{\text {heiix }(\theta)}\right]
\end{aligned}
$$

where

$$
\begin{aligned}
& {[\mathrm{S}]_{\text {sphere }}=\left[\begin{array}{ll}
1 & 0 \\
0 & 1
\end{array}\right]} \\
& {[\mathrm{S}]_{\text {diplane( } \theta)}=\left[\begin{array}{cc}
\cos 2 \theta & \sin 2 \theta \\
\sin 2 \theta & -\cos 2 \theta
\end{array}\right]} \\
& \cdot \mathrm{S}]_{\text {helix }(\theta)}=\frac{1}{2} e^{\mp j 2 \theta}\left[\begin{array}{cc}
1 & \pm j \\
\pm j & -1
\end{array}\right]
\end{aligned}
$$

$\varphi$ is the absolute phase of the matrix, $\varphi_{s}$ is a relative phase difference, $\theta$ is the orientation angle of the diplane and the helix around the line of sight, while $k_{s}, k_{d}$, and $k_{h}$ are the real parameters representing the three scattering mechanisms.

An important implication of such a decomposition is that scatterers of different nature may be completely and coherently separated even if they are within the same resolution cell of an image. In this sense, polarimetric processing effectively enhances the resolution capabilities.

Three-component decompositions like the above are particularly suited for the generation of color imagery, since the three components may be used for controlling the basic colors in RGB (Red, Green, Blue) images. Hence, it is possible in one image to reflect not only the true effective strength of the scattering, but also the nature of the scattering. 

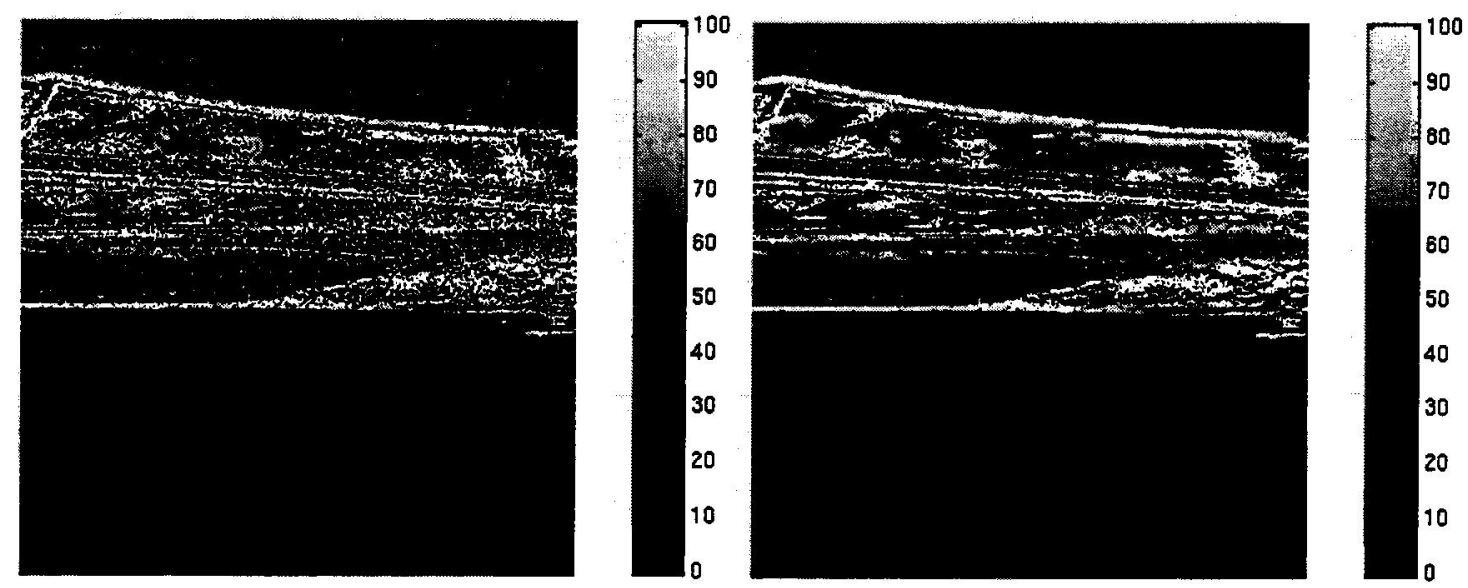

Figure 1: Results obtained processing the amplitude of the images. (a) Original $|\mathrm{Shh}|$ of the island image. (b) $|\mathrm{Shh}|$ processed of the same image.

\section{METHOD FOR SPECKLE REDUCTION}

Speckle reduction is important in the treatment of data from polarimetric SAR systems. One of the main applications of this kind of information is terrain classification, for which several types of classification methods are available. The performance of these techniques can be improved removing the speckle both from the amplitude and the phase of the data.

First of all, the wavelet techniques were applied to the amplitude information. In this case, classical techniques were applied, being based on transform coefficients thresholding. The thresholding can be done in two ways: through the Donoho method or through the Sigmoid method [9]. As can be seen in the Fig. 1, there is a clear speckle reduction and almost no loss of resolution.

The second step in the process is to remove speckle from phase information. In this case the process is different from that used in the case of amplitude information. An additive noise model is assumed instead the multiplicative one used on the amplitude. As will be shown afterwards, the assumption of an additive model is quite close to reality.

The scattered waves by a non-stationary target, in the case of polarimetric systems, cannot be fully characterised by the scattering matrix [S], so other kind of operators have to be used to have a complete characterisation [7]. These operators are the coherency matrix [T], the covariance matrix [C] and the Mueller matrix [M]. Through the vector expression of the scattering matrix $[\mathrm{S}], \overrightarrow{\mathrm{k}}$, the covariance matrix [C] can be obtained as:

$$
\begin{aligned}
& \overline{\mathbf{k}}=\left(\begin{array}{llll}
S_{h h} & S_{h v} & S_{v h} & S_{v v}
\end{array}\right)^{T} \text { where } S_{a b}=\vec{E}_{b} / \vec{E}_{a} \\
& {[\mathrm{C}]=\vec{k} \cdot \vec{k}^{T^{*}}}
\end{aligned}
$$

As each of the elements of $\vec{k}$ is complex, the covariance matrix [C] is complex too, so it can be written in the following way:

$$
[\mathbf{C}]=[\mathbf{X}]+j \cdot[\mathbf{Y}]
$$

Each of the elements of [C], $c_{i j}$, has a real part $x_{i j}$ and an imaginary part $y_{i j}$. These two parts can be represented by the expressions:

$$
\begin{aligned}
& x_{i j}=\operatorname{Re}\left(c_{i j}\right)=\left|S_{k l}\right| \cdot\left|S_{m n}\right| \cdot \cos \left(\phi_{r}\right) \\
& y_{i j}=\operatorname{Im}\left(c_{i j}\right)=\left|S_{k l}\right| \cdot\left|S_{m n}\right| \cdot \sin \left(\phi_{r}\right)
\end{aligned}
$$

where

$$
\begin{aligned}
& k, l, m, n \in h, v \\
& \phi_{r}=\phi_{k l}-\phi_{m n}
\end{aligned}
$$

Removing speckle from the matrix [C], is the same as to find an estimator of the matrix [C] without speckle. This task can be reduced to find the estimator of the real and imaginary parts of the $c_{i j}$ components (5). These estimators are: 
TABLE I

\section{NUMERICAL RESULTS OF THE PHASE IMAGES. SP INDICATES THE ORIGINAL IMAGE OR SPECKLED IMAGE WHEREAS USP INDICATES THE PROCESSED IMAGE OR UNSPECKLED IMAGE}

\begin{tabular}{|c|c|c|c|c|c|c|}
\hline & \multicolumn{2}{|c|}{ Lag o on thago } & \multicolumn{2}{|c|}{ Thland warge } \\
\hline & & & Mean & $\sigma$ & Mean & $\sigma$ \\
\hline \multirow{4}{*}{$\begin{array}{c}\text { Co-polar } \\
\text { phase } \\
\phi_{c}\end{array}$} & \multirow[b]{2}{*}{$\cos \left(\phi_{c}\right)$} & $S p$ & 0.5184 & 0.5955 & 0.5811 & 0.5892 \\
\hline & & Usp & $\overline{34}$ & 0.2251 & 0.5811 & 0.1577 \\
\hline & \multirow[b]{2}{*}{$\sin \left(\phi_{c}\right)$} & $\mathrm{Sp}$ & -0.0 & 0.6 & 0.0519 & 0.5590 \\
\hline & & Usp & -0.0 & 0.1834 & 0.0519 & 0.1031 \\
\hline \multirow{4}{*}{$\begin{array}{c}\text { Cross-polar } \\
\text { Phase } \\
\phi_{x}\end{array}$} & \multirow[b]{2}{*}{$\cos \left(\phi_{x}\right)$} & Sp & 0.0229 & 0.7043 & 0.0503 & 0.7415 \\
\hline & & Usp & 0.0229 & 0.1773 & 0.0503 & 0.1039 \\
\hline & \multirow{2}{*}{$\sin \left(\phi_{x}\right)$} & $S p$ & -0.0728 & 0.7058 & 0.0182 & 0.6688 \\
\hline & & Usp & -0.0728 & 0.1834 & 0.0182 & 0.0878 \\
\hline
\end{tabular}

$\bar{x}_{i j}=E\left\{x_{i j}\right\}=E\left\{\left|S_{k l}\right| \cdot\left|S_{m n}\right|\right\} \cdot E\left\{\cos \left(\phi_{r}\right)\right\}$

$\bar{y}_{i j}=E\left\{y_{i j}\right\}=E\left\{\left|S_{k l}\right| \cdot\left|S_{m n}\right|\right\} \cdot E\left\{\sin \left(\phi_{r}\right)\right\}$

Due to the fact that amplitude information is statistically independent from the phase information [10], the process can be reduced to finding an estimator of the amplitude information and an estimator of the phase information.

In a monostatic polarimetic system, the cross-polar components $S_{h v}$ and $S_{v h}$ are equal, and this fact produces that there are only two kind of phases $\phi_{\mathrm{r}}$ in equations (5) an (6). Theses phases are called co-polar phase $\phi_{c}$ and cross-polar phase $\phi_{x}$. The co-polar phase is $\phi_{c}-\phi_{v v}-\phi_{h h}$, and has an approximately Gaussian statistics [10], on the other hand, the expression of the cross-polar phase is $\phi_{\mathrm{x}}=\phi_{\mathrm{hv}}-\phi_{\mathrm{hh}}=\phi_{\mathrm{vh}}-\phi_{\mathrm{hh}}$, that has a uniform statistic over $[-\pi, \pi]$ [10]. The process proposed to remove speckle from phase information, do not process the phases $\phi_{\mathrm{c}}$ and $\phi_{\mathrm{x}}$ directly, instead of this the cosine and the sine of these phase are processed. At the same way as amplitude information, wavelet tools are applied but assuming an additive noise model, over the phase information. The process of speckle removal can be summarised in these points:
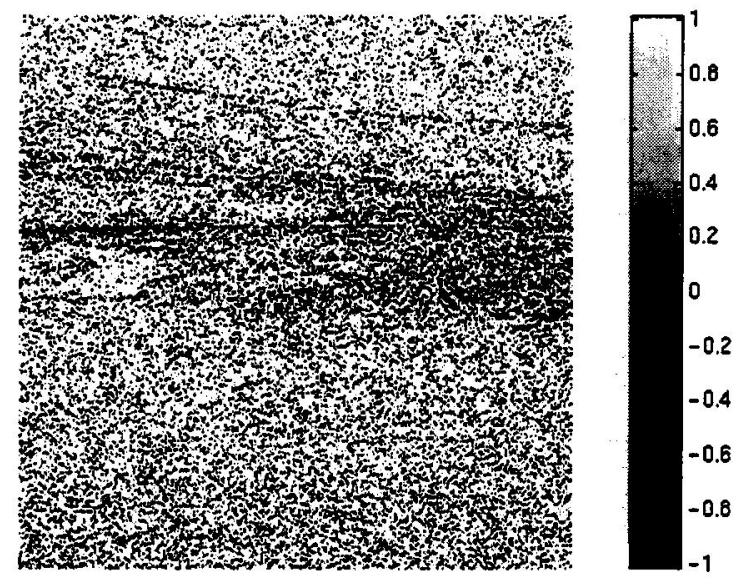

- Obtaining the function $\cos \left(\phi_{\mathrm{r}}\right)$ y $\sin \left(\phi_{\mathrm{r}}\right)$, where $\phi_{\mathrm{r}}=\phi_{\mathrm{c}}$ or $\phi_{\mathrm{r}}=\phi_{\mathrm{x}}$.

- Applying the Discrete Wavelet Transform or DWT to the previous function. In this case an additive noise model is assumed.

- Applying the methods of noise removing based on WT, Donoho method or Sigmoid method [9].

- Applying the Inverse Discrete Wavelet Transform IDWT to obtain the unspeckled information.

A good estimator of [C] can be obtained processing both the amplitude information and the phase information. At the same time, once the coherency matrix has been obtained the estimator of the Mueller matrix or coherency matrix can be calculated [4].

\section{RESULTS}

The previous algorithm has been applied to both, simulated images and real ones. The real images are Cband images taken by a mission of the Danish EMISAR. To test the performance of the algorithm, two kinds of images were taken. The first shows a kind of lagoon or marsh close to the sea, and the second shows one island of the Baltic Sea. TABLE I shows the numerical results of the phase images.

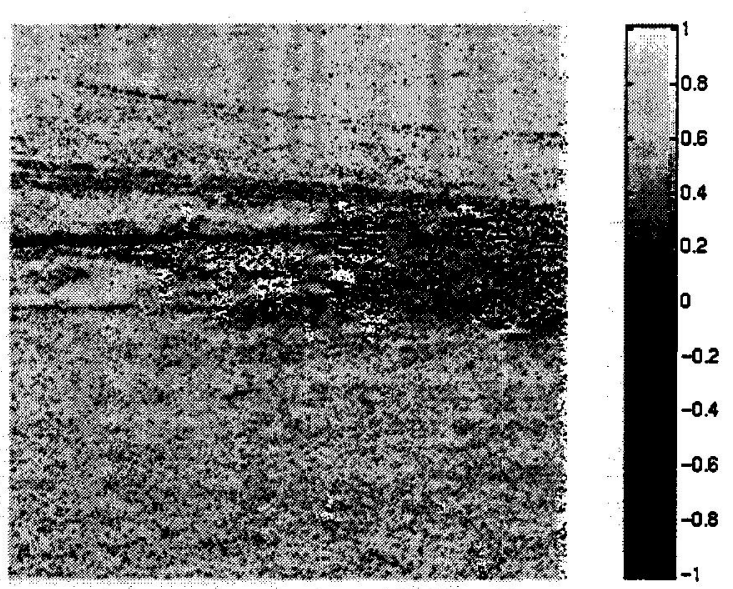

Figure 2: Processed phase images. Left (a): Original $\cos \left(\phi_{c}\right)$ of island image. Right (b): Processed $\cos \left(\phi_{c}\right)$ of same. 


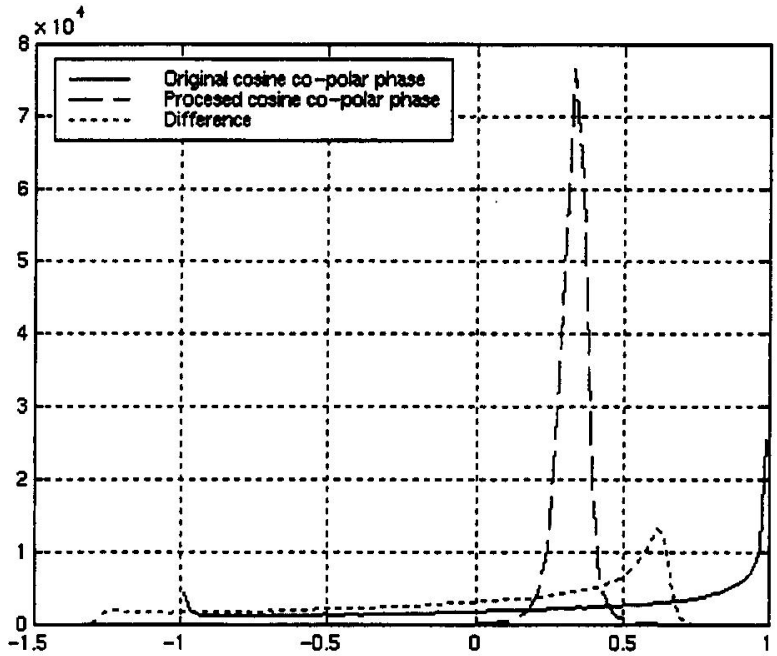

Figure 3: Histograms of a simulated co-polar phase. In this case the histograms of the original and processed cosine of the co-polar phase are shown. Also the difference between them is shown, this difference is the speckle noise.

In TABLE I different features of the processing systems can be seen. First of all, the process does not change the mean of the original image, which is very important overall in the field of classification. The second fact is that the process reduces the standard deviation of the image, with the consequent reduction of speckle noise. Another important aspect is that the image does not have a loss of resolution, as appears from the images. Fig. 2 shows the results obtained with the cosine of the co-polar phase of the island image. As can be seen, there is an obvious speckle reduction, and almost no loss of resolution.

The main reason to process the cosine and the sine of the phase information instead the phase information alone is that this information appears always in the operators $[\mathbf{C}],[\mathbf{M}]$ and $[\mathbf{T}]$ into cosine or sine functions. It has been seen that the cosine and the sine accentuate the frequencial separation between original data and noise. In this way the noise removing process, based on wavelet transform, can take advantage of this separation. Also in this process, an additive noise mode is assumed in the cosine and the sine of the phase information. To justify this model a simulation has been done. In this case an image simulates the co-polar phase $\phi_{c}$. In Fig. 3 three histograms are shown (original image -cosine of the copolar phase-, processed image and difference). In this case the processed image tends to a delta in the mean value of the original image ( 0.32$)$, just it would be expected in an additive model.

\section{CONCLUSIONS}

In this paper, some techniques for processing polarimetric SAR have been considered. In particular, a new way to remove speckle in polarimetric images was presented. This new algorithm is based on wavelet transform, making use of the advantages of this new transform. The wavelet transform with the Donoho or Sigmoid methods allows the reduction of speckle noise both from the amplitude and the information phase, with almost no loss of resolution.

\section{ACKNOWLEDGMENTS}

This work has been supported in part by a grant given to Carlos López by the Generalitat de Catalunya and also by the Spanish Commission for Science and Technology CICYT-TIC 96-0879, and by the European Commission under the programme for Training and Mobility of Researchers (TMR). Thanks are due to the Danish Center for Remote Sensing (DCRS), Department of Electromagnetic Systems, Technical University of Denmark, for making EMISAR data available.

\section{REFERENCES}

[1] Curiander, RC.: 'Synthetic Aperture Radar', John Wiley \& Sons Inc., NY, USA, 1991)

[2] Novak, L.M and M.C. Burl, 'Optical Speckle Reduction in Polarimetric SAR Imagery', IEEE Trans. Aerospace Electron. Sys., Vol. 26, 293-305, Mar. 1990.

[3] Lee J.S., M.R Grunes and S.A. Mango, 'Speckle Reduction in Multipolarisation Multifrequiency SAR imagery', IEEE Trans. Geosci. Remote Sensing, July 1991, Vol. GE-29.

[4] S. Goze and A. Lopes, 'A MMSE Speckle Filter for Full Reduction SAR Polarimetric Data', Joumal of Electromagnetic Waves and Applications, 1993, Vol. 7, No. 5, pp. 717-737.

[5] Krogager, E., 'Aspects of Polarimetric Radar Imaging', Doctoral Thesis, Technical University of Denmark, May 1993 (Danish Defence Research Establishment, P.O.Box 2715, DK-2100 Copenhagen, Denmark).

[6] Boerner, W.-M, et al., 'Polarimetry in Radar Remote Sensing: Basic and Applied Concepts', Chapter 5 in RA. Reyerson, ed., The Manual of Remote Sensing, 3rd ed.. ASPRS Publishing, Bethesda, MD, 1997.

[7] Cloude, S.R., and E. Pottier, 'A Review of Target Decomposition Theorems in Radar Polarimetry', IEEE Transactions on Geoscience and Remote Sensing, 34, 2, 498-518, Mar. 1996.

[8] Krogager, E. and Z.H. Czyż, 'Properties of the Sphere, Diplane, Helix Decomposition', Proc. Journées Internationales de la Polarimétrie Radar, JIPR'95, pp. 106-114, Nantes, France, 21-23 Mar., 1995.

[9] Sveinsson J.R and J.A. Benediktsson: 'Speckle reduction and enhancement of SAR images in the wavelet domain', IGARRS'96, Lincoln, Nebraska (USA), May 1996, pp. 63-66.

[10] Sarabandi K.: 'Derivation of phase statistics from the Mueller matrix'. Radio Science, 1992, 27, (5), pp. 553-560. 